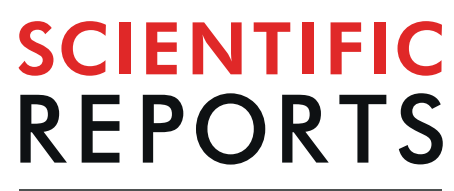

natureresearch

\title{
OPEN A SUMO-dependent pathway controls elongating RNA Polymerase II upon UV-induced damage
}

\begin{abstract}
Irina Heckmann ${ }^{1}$, Maximilian J. Kern ${ }^{1}$, Boris Pfander $^{2 *}$ \& Stefan Jentsch ${ }^{1,3}$
RNA polymerase II (RNAPII) is the workhorse of eukaryotic transcription and produces messenger RNAs and small nuclear RNAs. Stalling of RNAPII caused by transcription obstacles such as DNA damage threatens functional gene expression and is linked to transcription-coupled DNA repair. To restore transcription, persistently stalled RNAPII can be disassembled and removed from chromatin. This process involves several ubiquitin ligases that have been implicated in RNAPII ubiquitylation and proteasomal degradation. Transcription by RNAPII is heavily controlled by phosphorylation of the C-terminal domain of its largest subunit Rpb1. Here, we show that the elongating form of Rpb1, marked by $\mathrm{S} 2$ phosphorylation, is specifically controlled upon UV-induced DNA damage. Regulation of S2phosphorylated Rpb1 is mediated by SUMOylation, the SUMO-targeted ubiquitin ligase SIx5-SIx8, the Cdc48 segregase as well as the proteasome. Our data suggest an RNAPII control pathway with striking parallels to known disassembly mechanisms acting on defective RNA polymerase III.
\end{abstract}

DNA is the macromolecule that harbors all information required for life. In eukaryotes three nuclear RNA polymerases (RNAPI-III) are necessary to read out this information. While RNAPI transcribes ribosomal RNA $(\mathrm{rRNA})^{1}$, RNAPIII mediates transcription of rRNA, transfer RNAs (tRNA) and other small RNAs ${ }^{2}$. RNAPII is important for transcription of protein-coding genes, as well as for synthesis of non-coding RNAs ${ }^{3-5}$.

All three RNA polymerases are under control of several posttranslational modifications. However, specifically transcription by RNAPII is regulated by phosphorylation that chiefly targets Rpb1's carboxyl-terminal domain (CTD) heptapeptide repeats (consensus sequence YSPTSPS ${ }^{6}$, which serve as platform for factors involved in transcription, chromatin modification, mRNA splicing and export ${ }^{7,8}$. Phosphorylation of serine-5 (S5P) of the CTD repeats appears to be largely specific for Rpb1 molecules localized to promoters ${ }^{9,10}$. Serine-2 phosphorylation (S2P) in contrast is a hallmark for elongating polymerases and ChIP signals using S2P-specific antibodies steadily rise downstream of transcription start sites and culminate at the 3 '-ends of genes ${ }^{9,11-13}$.

Highly problematic for all RNA Polymerases are bulky DNA lesions on the coding strand as they may behave as "roadblocks" leading to persistent stalling of the progressing protein complexes. A key mechanism to remove bulky DNA adducts and to avoid cell cycle arrest and cell death is nucleotide excision repair (NER) ${ }^{14,15}$. Yet to conduct DNA repair the machinery first needs to gain access to the lesion that is masked by a stalled polymerase and here, several possibilities exist. First, the RNA polymerase can simply be released from the DNA to allow access. This has been shown to occur for RNAPI and RNAPII, but the underlying mechanism is poorly understood $^{16-18}$. Second, RNAPII backtracking from the lesion can facilitate repair without dissociation. This is made possible by the proofreading activity of RNAPII, which allows the complex to backtrack during transcription ${ }^{19,20}$. After RNAPII backtracking and successful DNA repair, reactivation of transcription requires RNA transcript cleavage for repositioning of the $3^{\prime}$-end with the active center of RNAPII ${ }^{21,22}$. Finally, active removal or even degradation of the stalled polymerase may serve as a temporary relief and perhaps facilitate DNA repair ${ }^{23-25}$.

Previous work has demonstrated that in cells exposed to UV light, which leads to pyrimidine dimers and 6-4 photoproducts in DNA, Rpb1, the largest subunit of RNAPII, becomes short-lived through degradation by the ubiquitin-proteasome system (UPS) ${ }^{26}$. By monitoring the entire population of Rpb1 using Rpb1-specific

${ }^{1}$ Max Planck Institute of Biochemistry, Molecular Cell Biology, 82152, Martinsried, Germany. ${ }^{2}$ Max Planck Institute of Biochemistry, DNA Replication and Genome Integrity, 82152, Martinsried, Germany. ${ }^{3}$ Stefan Jentsch is deceased.

*email: bpfander@biochem.mpg.de 
antibodies, it was initially shown that Rpb1 is ubiquitylated by the ubiquitin ligase Rsp5, which binds to the CTD of Rpb1 ${ }^{27-31}$. Other studies found that Rpb1 ubiquitylation is mediated by an Elongin-Cul3-dependent ubiquitin ligase complex (Cul3-Roc1-Elc1-Ela1) ${ }^{32,33}$. Later, it was suggested that the two distinct ubiquitin ligases act within a sequential pathway ${ }^{23,34}$. In the current model, Rpb1 is first mono-ubiquitylated or modified by a lysine- 63 (K63)-linked poly-ubiquitin chain. The de-ubiquitylation enzyme Ubp2 removes Rsp5-dependent K63-linked poly-ubiquitin chains to mono-ubiquitylation, allowing the Elongin-Cul3 ligase to add K48-linked poly-ubiquitin chains that induce Rpb1 degradation ${ }^{34}$. Another de-ubiquitylation enzyme, Ubp3, was suggested to reverse both Rpb1 poly- and mono-ubiquitylation ${ }^{35}$. Removal of K48-linked poly-ubiquitylated Rpb1 from damaged DNA is furthermore dependent on the ATPase $\mathrm{Cdc} 48^{36}$. To this end, Cdc48 cooperates with the adaptor proteins Ubx4/ Ubx 5 to mediate extraction of Rpb1 from the chromatin-bound RNAPII complex and subsequent delivery to the proteasome for degradation ${ }^{36,37}$.

Apart from the above-mentioned negative regulation by ubiquitin, the catalytic core domain of Rpb1 is also modified with SUMO, which requires the SUMO ligase Siz1 together with the SUMO-conjugating enzyme $\mathrm{Ubc}^{38,39}$. SUMOylation of Rpb1 is induced upon DNA damage or transcriptional impairment, but has not been linked to Rpb1 ubiquitylation or proteasomal degradation. Instead, this modification is thought to restrain DNA damage signaling induced by a stalled RNAPII complex ${ }^{39}$.

In this study, we revealed a further layer of RNAPII regulation upon DNA damage in the budding yeast Saccharomyces cerevisiae. Focussing on the transcription-engaged pool of RNAPII we discovered a SUMO-dependent mechanism, which specifically controls the serine-2 phosphorylated (S2P) form of RNAPII. Upon UV treatment, we observed rapid SUMOylation of Rpb1-S2P and subsequent decrease of both S2P- and SUMO-modified Rpb1. Disappaerance of S2P-modified Rpb1 is dependent on the SUMO-targeted ubiquitin ligase Slx5-Slx8, the ubiquitin/SUMO- dependent segregase Cdc48 and the proteasome.

\section{Results and Discussion}

S2-phosphorylated Rpb1 declines upon DNA damage induction via a mechanism involving the proteasome and Cdc48 segregase. The fate of transcriptionally stalled RNAPII upon DNA damage has been investigated previously ${ }^{26,28-30,34}$. Degradation of the RNAPII largest subunit Rpb1 has been shown using antibodies recognizing the entire Rpb1 pool (such as monoclonal antibodies $4 \mathrm{H} 8$ and $8 \mathrm{WG} 16)^{26,34,40}$. As the fate of elongating RNAPII, which would collide with DNA lesions is particularly relevant, we aimed to monitor the S2-phosphorylated form as a proxy for elongating RNAPII (monoclonal antibody 3E $10^{9,40-42}$ ). To assay RNAPII levels, we challenged budding yeast cells with an acute dose of UV light $\left(400 \mathrm{~J} / \mathrm{m}^{2}\right)$ and probed samples at different time points after irradiation by western blotting using the different antibodies. Using antibodies against the entire pool of Rpb1 (4H8 and 8WG16), we observed a moderate loss of Rpb1 signals over 4 hours indicating degradation $^{26,27,34,43}$ (Fig. 1A, left and central panel and Fig. 1B). When we used the S2P-specific antibody (3E10) to probe these samples, we observed that the Rpb1 S2P signal started to decrease $2 \mathrm{~h}$ after UV treatment and dropped almost by fivefold within $4 \mathrm{~h}$ after UV treatment (Fig. 1A, right panel and Fig. 1B). In contrast, UV treatment had no discernable effect on the Rpb1-S5P signal (Fig. S1A, right panel). Although we followed the same Western Blot densiometry protocol in all experiments, it needs to be noted that effects observed with different antibodies might be difficult to compare in quantitative manner. Nonetheless, we found striking that specifically the elongating, S2-phosphorylated form of RNAPII underwent strong changes after UV treatment.

To further analyze whether the drop in Rpb1-S2P levels was due to protein turnover, we treated cells with the protein synthesis inhibitor cycloheximide with or without additional induction of UV damage. While in cycloheximide-treated cells the Rpb1-S2P turnover was moderate (Fig. S1B, left panel), the Rpb1-S2P signal dropped much faster, when a combination of CHX and UV treatment was used (Fig. S1B, right panel). A UV dose of $400 \mathrm{~J} / \mathrm{m}^{2}$ is lethal for the majority of cells, but UV-induced reduction in the Rpb1-S2P signal occurred in a dose-dependent manner over a wide range of UV doses $\left(50 \mathrm{~J} / \mathrm{m}^{2}-400 \mathrm{~J} / \mathrm{m}^{2}\right.$, Fig. S1C-E).

We also used the UV-mimetic compound 4-nitroquinoline 1-oxide (4NQO) ${ }^{44}$, which induces DNA damage through reactive oxygen species that is also repaired by nucleotide excision repair. Also treatment of yeast cells with $20 \mu \mathrm{g} / \mathrm{ml} 4$ NQO caused a decrease in the Rpb1-S2P signal (Fig. S1F).

Previous work has demonstrated that exposure to UV or 4NQO leads to degradation of Rpb1 by the ubiquitin-proteasome system (UPS) ${ }^{26,27,34}$. Therefore we wanted to clarify, whether the observed loss of the Rpb1-S2P signal was also dependent on the proteasome. Indeed, the Rpb1-S2P signal remained stable when proteasome mutant cim3-1 cells were treated with UV light (Fig. 1C). Moreover, when we tested for involvement of the ubiquitin/SUMO-dependent segregase Cdc48 $8^{36,45,46}$, we found that the Rpb1-S2P signal was stabilized in UV-challenged $c d c 48-6$ and $c d c 48-3$ mutant cells (Fig. 1D). Overall, these data suggest that the elongating, S2-phosphorylated pool of RNAPII is diminished after DNA damage in a manner that depends on the proteasome and Cdc48.

UV damage triggers Ubc9-, Siz1- and Siz2-dependent SUMOylation of Rpb1. After UV irradiation, we consistently observed a slower migrating Rpb1 species that reacted with all Rpb1 antibodies used, suggesting that this was a post-translationally modified version of Rpb1. Levels of this modified Rpb1 species were especially pronounced at early time points after UV exposure (Fig. 1A). Several RNAPII subunits were previously reported to be SUMO substrates ${ }^{47,48}$ and Rpb1 specifically becomes SUMOylated ${ }^{38,39,48,49}$. We therefore tested whether the observed slower migrating species is a SUMOylated form of Rpb1. We expressed a variant of SUMO (Smt3 in yeast) fused with an N-terminal GFP-tag (GFP-SUMO). Expression of GFP-SUMO caused an upshift of the slower migrating species of Rpb1 (Fig. 2A), suggesting it was indeed SUMO-modified Rpb1. To confirm this finding, we also probed immunoprecipitated Rpb1 from unchallenged and UV-treated cells by western blotting with a SUMO-specific antibody and found that the upshifted Rpb1 species after UV damage contained SUMO (Fig. 2B, WT + UV). 
A

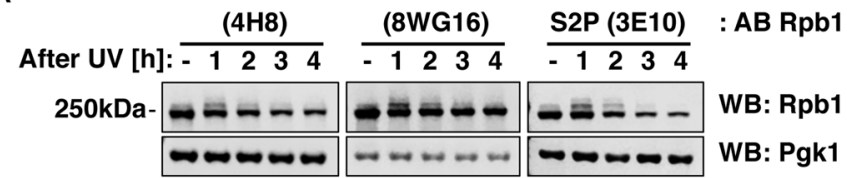

B

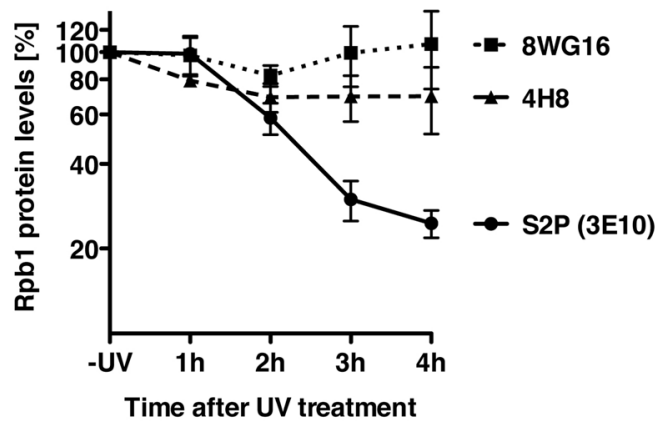

C

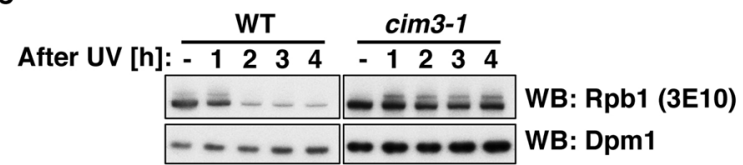

D

\begin{tabular}{|c|c|c|c|c|c|}
\hline \multirow[b]{2}{*}{ After UV [h] } & WT & $c d c 48-6$ & WT & cdc48-3 & \\
\hline & $\begin{array}{l}-1234 \\
\end{array}$ & $\begin{array}{l}-1234 \\
\end{array}$ & -12234 & -1234 & \\
\hline & - & - - - - - & $=-$ & $=---$ & WB: Rpb1 (3E10) \\
\hline & $-\cdots-\cdots$ & $-\cdots-\cdots$ & $--n-\cdots$ & $-\cdots-\cdots$ & WB: Dpm1 \\
\hline
\end{tabular}

Figure 1. S2-phosphorylated Rpb1 disappears upon DNA damage induction via a mechanism involving the proteasome and the segregase Cdc48. (A) Levels of Rpb1 and modified forms in WT cells treated with UV irradiation $\left(400 \mathrm{~J} / \mathrm{m}^{2}\right)$ followed by a recovery time course in YPD media in the dark, which blocks repair by photolyase. For the western blots (WB) antibodies against the C-terminal domain (CTD) of Rpb1 were used. $3 \mathrm{E} 10$ recognizes phosphorylated serine-2 in the CTD ${ }^{9,13,41}, 4 \mathrm{H} 8$ and 8WG16 recognize the CTD independently of any modification ${ }^{82,83}$. Pgk1 served as loading control. (B) Quantification of Rpb1 signals as in (A). Quantification was performed on LI-COR Odyssey with normalization to Pgk1. Data represent mean \pm standard deviation calculated from four biological replicates, presented as relative amount to the untreated (-UV) sample. (C+D) Rpb1-S2P levels in WT, proteasome mutant cim3-1 (C) and $c d c 48-6$ or $c d c 48-3$ (D) cells after UV irradiation $\left(400 \mathrm{~J} / \mathrm{m}^{2}\right)$ followed by a recovery time course in YPD media. Cells were shifted to $37^{\circ} \mathrm{C}$ for $1 \mathrm{~h}$ before irradiation and for the recovery phase. For the western blots (WB) anti-Rpb1 (3E10) antibody was used. Dpm1 served as loading control.

Next, we introduced mutations into the SUMO pathway. Using mutants of the SUMO-conjugating enzyme Ubc9 and the SUMOligases (Siz1 and Siz2), we corroborated previous findings ${ }^{39,48}$ and found that Rpb1 SUMOylation is mediated by Ubc9, Siz1, and to a lesser extent by Siz2 (Fig. 2B, right panel). However, we still observed Rpb1 SUMOylation when we mutated a proposed target lysine residue (K1487) ${ }^{39}$, as well as several other potential target sites ${ }^{31}$ to non-SUMOylatable arginine residues (Fig. S2). These data are consistent with the idea that SUMO may target multiple lysine residues of Rpb1, as has been seen for other SUMO substrates ${ }^{47,49}$.

Serine-2 phosphorylated Rpb1 is regulated by a SUMO-dependent pathway. To investigate whether UV-induced Rpb1 SUMOylation and the decline of the Rpb1-S2P signal are related, we investigated UV-induced loss of S2-phosphorylated Rpb1 in SUMO pathway mutant cells. Strikingly, the Rpb1-S2P signal was stable in mutants defective in Ubc9 or Siz1 even after UV irradiation (Fig. 3A,B). In contrast, SIZ2-deficient cells showed loss of the Rpb1-S2P signal, similar to WT cells (Fig. 3B). The same set of enzymes, which are responsible for UV-induced Rpb1 SUMOylation are therefore required for UV-induced disappearance of S2-phosphorylated Rpb1. Overall, these data are consistent with several models: (i) the SUMO-dependent mechanism acts on regulators of elongating RNAPII like the kinases relevant for S2 phosphorylation. (ii) UV-induced SUMOylation of Rpb1 triggers removal of stalled RNAPII from chromatin or renders it otherwise resistant to S2 phosphorylation. (iii) The SUMO-dependent mechanism leads to degradation of Rpb1 from stalled RNAPII complexes.

To address the first model, we tested whether the kinases that mediate CTD S2 phosphorylation of Rpb1 ${ }^{13,50,51}$ are degraded after UV damage. We used antibodies against $\mathrm{Ctk} 1^{52}$ or Bur $1^{53}$ and followed respective protein levels after UV treatment (Fig. S3A,B). Interestingly, however, we found that endogenous protein levels of Ctk1 and Bur1 did not change significantly after UV treatment, while Rpb1-S2P levels dropped (Fig. S3A,B). Therefore, 


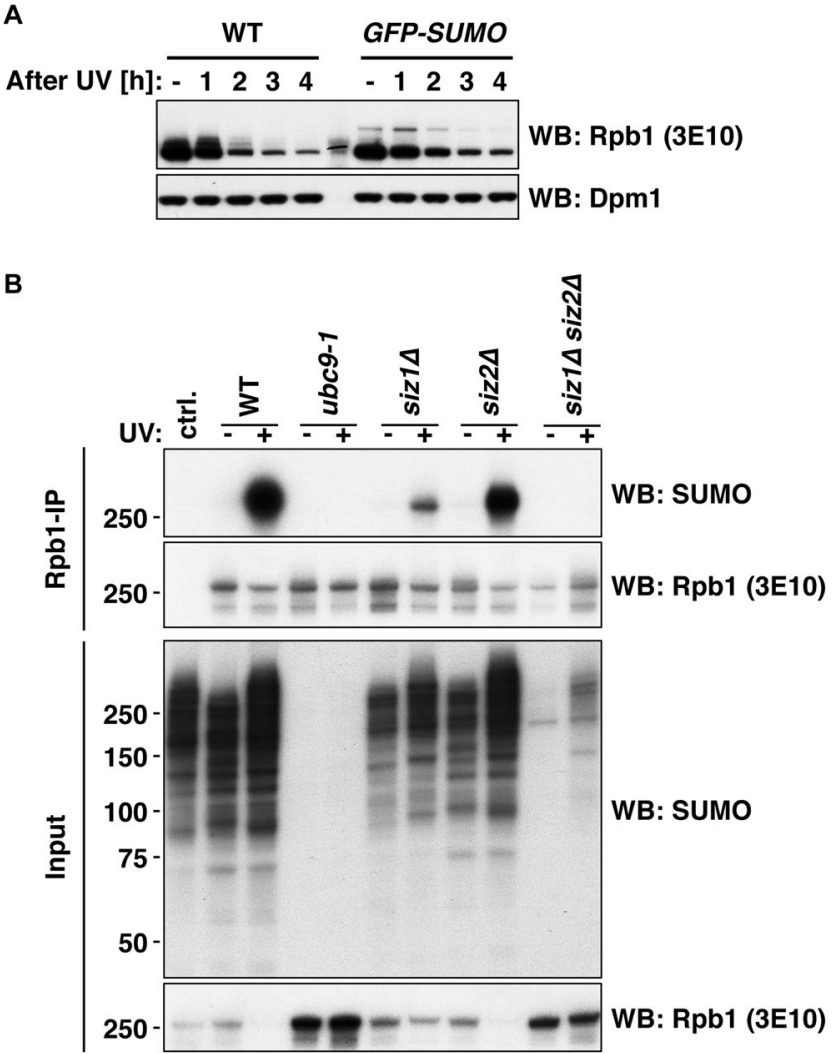

Figure 2. Rpb1 of elongating RNAPII is SUMOylated upon UV treatment. (A) Rpb1-S2P and the corresponding SUMO-modified form in WT cells and cells expressing GFP-tagged SUMO after UV irradiation $\left(400 \mathrm{~J} / \mathrm{m}^{2}\right)$ followed by a recovery time course in YPD medium. SUMOylation is indicated by mobility shift of Rpb1-S2P with the tagged SUMO protein. The 3E10 antibody was used to detect Rpb1. Dpm1 served as loading control. (B) Immunoprecipitation of Rpb1 with Rpb1-S2P-specific antibody (3E10) from UV-treated and untreated WT, $u b c 9-1$, siz1 $\Delta$, siz2 $\Delta$ and siz1 $\Delta$ siz2 $\Delta$ double mutant cells. SUMOylated species of Rpb1 were detected by western blotting (WB) using SUMO-specific antibody.

A

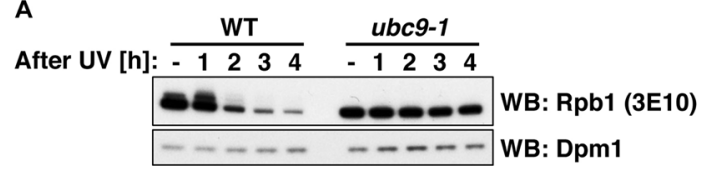

B

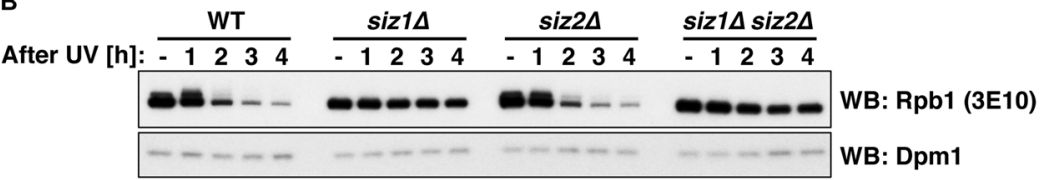

Figure 3. The SUMO conjugating system is required for UV-induced loss of S2-phosphorylated Rpb1. (A+B) Levels of Rpb1-S2P in WT and $u b c 9-1$ cells (A) and siz1 $\Delta$, siz2 $\Delta$ and siz1 $\Delta$ siz2 $\Delta$ double mutant cells (B) after UV irradiation $\left(400 \mathrm{~J} / \mathrm{m}^{2}\right)$. In (A) cells were shifted to $37^{\circ} \mathrm{C}$ for $1 \mathrm{~h}$ before irradiation followed by a recovery in YPD media at $37^{\circ} \mathrm{C}$. The $3 \mathrm{E} 10$ antibody was used to detect Rpb1 by western blotting (WB). Dpm1 served as loading control.

the S2-phosphorylating kinases are apparently not regulated by UV, at least not on protein level. Yet, these experiments do not exclude that SUMO and ubiquitin regulate the S2-phosphorylated pool of Rpb1 by other means.

SUMO-targeted ubiquitin ligases (STUbLs) control RNAPII after UV damage. Similar to our findings, also RNA polymerase III was recently found to be a SUMOylation target and RNAPIII SUMOylation was found to be induced when RNAPIII transcription was defective ${ }^{54,55}$. Interestingly, SUMO modification led to RNAPIII ubiquitylation by the heterodimeric SUMO-targeted ubiquitin ligase (STUbL) Slx 5 -Slx $8^{55}$. This ubiquitin-modification was found to induce Cdc48-dependent extraction of RNAPIII from chromatin 
A

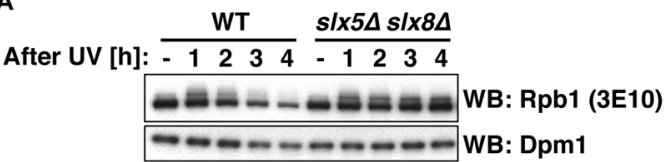

B
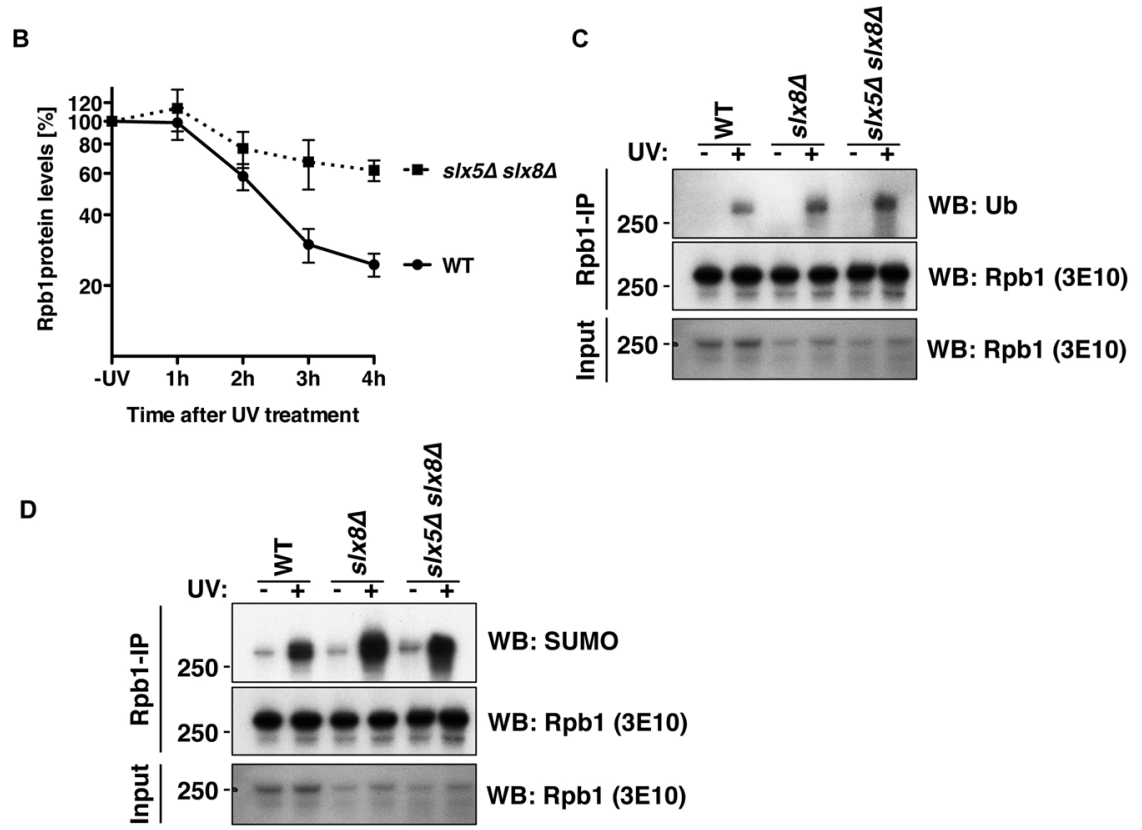

E

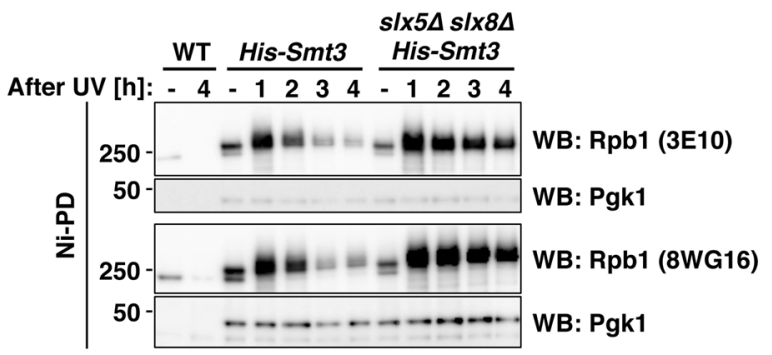

Figure 4. The STUbL Slx5-Slx8 regulates the SUMOylated pool of Rpb1. (A) Levels of Rpb1-S2P in WT and sl $x 5 \Delta$ sl $x 8 \Delta$ cells after UV irradiation $\left(400 \mathrm{~J} / \mathrm{m}^{2}\right)$. The $3 \mathrm{E} 10$ antibody was used to detect Rpb1 by western blotting (WB). Dpm1 served as loading control. (B) Quantification of Rpb1-S2P levels in WT and STUbLdeficient $\operatorname{sl} x 5 \Delta$ slx $8 \Delta$ cells as described in (A). Quantification was performed on a LI-COR Odyssey system with normalization to Pgk1. Shown are mean \pm standard deviation calculated from four biological replicates, presented as relative amount compared to the untreated $(-\mathrm{UV})$ sample. $(\mathrm{C}+\mathrm{D})$ Immunoprecipitation of Rpb1 with Rpb1-S2P-specific antibodies (3E10) from UV-treated and untreated WT, sl $x 8 \Delta$ and $s l x 5 \Delta s l \times 8 \Delta$ cells. Ubiquitylated (C) and SUMOylated (D) species of Rpb1 were detected by western blotting (WB) using ubiquitin- and SUMO-specific antibodies succesively on the same blot. Therfore, control blots for Rpb1 are the same for (C) and (D). (E) Denaturing Ni-NTA pulldowns (Ni-PD) were performed to isolate His-SUMOconjugates from UV-treated WT and sl $x 5 \Delta$ slx $8 \Delta$ cells following recovery in YPD. SUMOylated species of Rpb1 were detected by western blotting (WB) using 3E10 (Ser2P-CTD) or 8WG16 (CTD) antibodies. SUMOylated Pgk1 served as pulldown control.

and downregulation of RNAPIII transcription, most likely by proteasomal degradation ${ }^{55,56}$. Given the similarities to UV-induced regulation of RNAPII-S2P, we hypothesized that also RNAPII might be regulated by Slx5-Slx8. Indeed, we found that cells deficient in Slx5-Slx8 did not show a reduction in the Rpb1-S2P signal after UV-induced DNA damage, but rather the Rpb1-S2P signal remained stable (Fig. 4A,B).

We therefore asked whether Rpb1 is a ubiquitylation substrate of Slx5-Slx8 and probed Rpb1-S2P immunoprecipitated from unchallenged and UV-treated cells with ubiquitin-specific antibodies. UV-induced ubiquitylation of Rpb1-S2P was readily detectable (Fig. 4C). Notably, however, this ubiquitin modification was apparently unrelated to Slx5-Slx8 function, as we still observed an Rpb1-S2P ubiquitin signal in cells lacking SLX8 or both SLX5 and SLX8 (Fig. 4C). In contrast, similar to published data ${ }^{28,32,33}$, we found Rpb1 ubiquitylation to depend on the ubiquitin ligases Rsp5 and Elongin-Cul3 (Fig. S4A,B). Specifically, we probed for ubiquitylation of immunoprecipitated Rpb1-S2P using ubiquitin-specific antibodies after UV treatment and found it to be defective in a temperature-sensitive mutant of RSP5 (rsp5-1) (Fig. S4A) and in cells lacking subunits of the Elongin-Cul3 ligase 
(elc1 $\Delta$, ela1 $\Delta$ and cul3 $\Delta$ ) (Fig. S4B). Moreover, the Rsp5-dependent ubiquitylation signal does also not rely on the SUMO-conjugating enzyme Ubc9 or the SUMO ligases Siz1 and Siz2 (Fig. S4C,D). Overall, we thereby confirmed previous reports, showing that Rpb1 is ubiquitylated involving both Rsp 5 and the Elongin-Cul3 ligase complex $^{27,32,33}$, but this ubiquitylation signal is apparently unrelated to the SUMO-STUbL pathway. We therefore conceded the assay was unable to visualize STUbL-dependent ubiquitylation of Rpb1, suggesting that either (i) STUbL-dependent ubiquitylation of Rpb1 is too short-lived to be detected in our IP-based assay, (ii) that STUbLs target RNAPII on subunits other than Rpb1, similar to RNAPIII, where the primary ubiquitylation target of Slx5-Slx8 is Rpc $160^{55}$ or (iii) that STUbLs do not target RNAPII at all, but rather a protein involved in RNAPII phosphorylation or transcription elongation in general.

In either of the first two scenarios, SUMO-modified Rpb1-S2P could be seen as an intermediate on the way to ubiquitylation and this intermediate would be expected to accumulate in the absence of STUbLs. Consistent with this idea, we detected an accumulation of the SUMOylated form of Rpb1-S2P when we treated cells lacking SLX8 or both SLX5 and SLX8 with UV (Fig. 4D). To further support these data, we enriched SUMOylated proteins using denaturing Ni-NTA pulldown of His-SUMO conjugates. SUMOylated proteins from WT and STUbL-deficient cells before UV treatment and at different time points thereafter were enriched and probed with the Rpb1 S2P-specific antibody (3E10) or the whole pool antibody (8WG16) (Fig. 4E). In line with our previous findings, SUMOylated species of Rpb1 peaked one hour after UV treatment and dropped gradually thereafter. In SLX5-SLX8-deficient cells, however, loss of SUMOylated Rpb1 species was minor and delayed, independent of the antibody used for Rpb1 detection (Fig. 4E). Overall, we therefore conclude that Rpb1-SUMOylation and loss of the Rpb1-S2P signal are connected, either because (i) Rpb1-SUMO and Slx5-Slx8 mediate ubiquitylation and chromatin extraction of Rpb1 or (ii) Rpb1-SUMO and Slx5-Slx8 regulate another factor, which critically controls the phosphorylation of RNAPII or perhaps transcription elongation itself.

Our data therefore reveal an unexpected function of SUMO and the STUbL Slx5-Slx8 in the regulation of RNAPII after UV-induced DNA damage. Our genetic and biochemical data point to several possible molecular mechanisms that could be responsible for loss of Rpb1-S2P.

First, Rpb1-S2P loss might be caused by STUbL-dependent Rpb1 ubiquitylation and degradation. This model would be entirely consistent with the involvement of the proteasome and the STUbL-dependent stabilization of SUMOylated Rpb1. However, when testing with IPs we did not observe evidence of STUbL-dependent Rpb1-S2P ubiquitylation. Rpb1 might therefore not be a direct target for Slx5-Slx8-dependent ubiquitylation. Alternatively, the modification might be very transient, as it is often observed for proteolysis-inducing ubiquitylation marks ${ }^{55,57,58}$. Notably, we could confirm Rsp5- and Elongin-Cul3 complex-dependent ubiquitylation on $\mathrm{Rpb} 1^{28,32,33}$. One possible scenario is therefore that Rpb1 is targeted by different kinds of ubiquitin modifications. In this scenario the Rsp5/Elongin-Cul3-dependent ubiquitylation would not lead to degradation of the S2-phosphorylated polymerase and is therefore detectable in IP experiments while the Slx5-Slx8-dependent ubiquitylation would lead to degradation of the S2-phosphorylated polymerase and is therefore very transient. Overall, the relation between Rsp5/Elongin-Cul3 and STUbL-dependent mechanisms needs further clarification.

Second, Rpb1-S2P loss could come from turnover of the entire RNAPII complex, not just the Rpb1 subunit. SUMOylated Rpb1 might serve as a recruiting factor for the STUbL Slx5-Slx8, but then target RNAPII subunits other than Rpb1 would become ubiquitylated, potentially explaining why no STUbL-dependent ubiquitylation was detected for Rpb1. STUbL-dependent ubiquitylation of proteins that are in complex with the SUMOylated STUbL-recruitment factor have been suggested in other cases and importantly for RNAPIII ${ }^{55,59,60}$ giving precedence for this model. In contrast, previous work has shown that specifically Rpb1, but not other subunits become destabilized upon UV treatment ${ }^{61}$.

Third, UV-induced regulation of the Rpb1 kinases/phosphatases could lead to Rpb1-S2P loss. The major Rpb1-CTD S2 kinases are Ctk1 and Bur1, which belong to the cyclin-dependent kinase (CDK) family ${ }^{6,13,62}$. Besides RNAPII, they target also other factors to regulate several aspects of transcription ${ }^{63-65}$ and translation $^{52}$. Particularly, histone ubiquitylation and SUMOylation were shown to prevent Ctk1 recruitment to chromatin and subsequent Rpb1-S2 phosphorylation ${ }^{66,67}$. Histone SUMOylation could therefore be involved in SUMO-dependent downregulation of Rpb1-S2 after UV, but current data do not explain the involvement of Slx5-Slx8. Other possible ways how Ctk1 and Bur1 could be regulated after UV could involve the CDK-activating kinase Cak1 ${ }^{68,69}$ or the Fcp1 phosphatase, which dephosphorylates the CTD at serine $2^{50}$.

Fourth, Rpb1-S2P loss might simply reflect removal of RNAPII from chromatin by the Cdc48 segregase and regulation in a non-degradative fashion. However, this hypothesis in itself does not explain the involvement of the proteasome mediating decrease of the Rpb1-S2P signal. An involvement of the proteasome would therefore have to be indirect. A speculative scenario could be, that in proteasome mutants, extraction of RNAPII is linked to Hpr1, a member of the THO/TREX complex involved in mRNA export. Hpr1 is known to be regulated by the proteasome ${ }^{70,71}$. Degradation of Hpr1 after mRNA export is crucial to release chromatin-bound RNAPII ${ }^{72}$. Hpr1 was also shown to be SUMOylated, but SUMOylation does not appear to affect its stability, but rather interactions with $\mathrm{mRNPs}^{73}$.

Overall, although the precise mechanism by which STUbLs and the SUMO pathway regulate RNAPII after UV-induced DNA damage will need to be further clarified by future research, we now add another layer of regulation of RNAPII upon transcriptional stalling. Our data suggest that RNAPII is controlled by the sequential action of the small ubiquitin-like modifier (SUMO) and ubiquitin. Such a sequential mechanism allows the opportunity to differentially recruit proteins containing ubiquitin-and/or SUMO-interacting motifs. For instance, Cdc48 together with its co-factor Ufd1 were shown to target both ubiquitylated and SUMOylated proteins and, strikingly, also STUbL substrates ${ }^{46,60,74}$. SUMOylation of stalled RNAPII might therefore be a first line of defense and help in recruiting repair factors. Prolonged stalling may require additional means and involve Slx5-Slx8 and Cdc48, perhaps to segregate RNAPII from chromatin similar to its role in the release of chromatin-associated proteins or complexes $s^{5,60,75,76}$. 


\section{Materials and Methods \\ Yeast strains. Yeast strains used in this study are listed in Table S1.}

Yeast techniques and cloning. Yeast strains used in this study were constructed using standard techniques ${ }^{77,78}$. All strains are isogenic to DF5 (except for FW1808, which is derived from FY56 ${ }^{79}$ ) and have the genotypes as described in Table S1. Yeast His-tagged SUMO was expressed from the integrative vector YIplac211 under the control of the $A D H 1$ promoter. Rpb1 point mutant variants were constructed using standard mutagenesis PCR techniques and expressed from the centromeric YCplac111 under the endogenous RPB1 promoter.

UV-C treatment of yeast cells. Yeast cells were grown to an $\mathrm{OD}_{600}$ of 0.5 at $30^{\circ} \mathrm{C}$ in YPD media, after which they were optionally incubated for $1 \mathrm{~h}$ at $37^{\circ} \mathrm{C}$ to induce temperature-sensitive phenotypes. Otherwise, cells were grown to an $\mathrm{OD}_{600}$ of 1 at $30^{\circ} \mathrm{C}$. About $50 \mathrm{OD}_{600}$ of cells were harvested and washed once with PBS. Cells $\left(50 \mathrm{OD}_{600}\right.$ ) were pelleted, washed once with PBS and irradiated in $140 \mathrm{~mm}$ culture dishes (BS04-irradiation chamber, Dr. Gröbel UV-Elektronik $\mathrm{GmbH}$ ) in $40 \mathrm{ml}$ PBS buffer. The cell suspension was pelleted in a dark falcon tube ( $5 \mathrm{~min}, 500 \mathrm{~g}$ ) and resuspended in $30 \mathrm{ml} \mathrm{YPD}$. To follow UV-recovery, cells were incubated for $4 \mathrm{~h}$ at $30^{\circ} \mathrm{C}$ or $37^{\circ} \mathrm{C}$ while shaking. For protein analysis, samples of $0.8 \mathrm{OD}_{600}$ cells were harvested every hour, pelleted, frozen in liquid nitrogen and stored at $-80^{\circ} \mathrm{C}$. For cell survival analysis, cells were plated on YPD plates after 4 hours of recovery and cell colonies were counted after 2 days.

Protein analysis. Total cellular protein extracts were prepared by TCA precipitation ${ }^{77}$, separated using NuPAGE Bis-Tris 4-12\% gradient gels (Life Technologies) and analyzed by standard western blotting techniques.

Immunoprecipitation of Rpb1 and Ni-NTA pulldown. For Rpb1-immunoprecipitation, about $50 \mathrm{OD}_{600}$ cells were resuspended in lysis buffer (50 mM HEPES pH 7.5, $1 \mathrm{mM}$ EDTA, 1\% Triton X-100, 0.1\% Na-deoxycholate, $10 \mathrm{mM}$ NEM, and complete protease inhibitors). Cell lysis was performed with a beadshaker (MM301, Retsch) using silica beads. Afterwards, cell lysates were sonicated (water bath sonification: Bioruptor UCD-200, Diagenode) and centrifuged $(21,000 \mathrm{~g}, 10 \mathrm{~min})$. The extract was incubated with Rpb1 antibodies (3E10 or 8WG16) for 1.5h. Protein A Sepharose (GE healthcare) was added for $30 \mathrm{~min}$. Incubation steps were performed on a rotation wheel at $4^{\circ} \mathrm{C}$. The beads were pelleted, washed with lysis buffer (3-times) and boiled in Laemmli buffer.

Denaturing Ni-NTA pulldowns were described previously ${ }^{80}$. About $50 \mathrm{OD}_{600}$ of cells were harvested, frozen in liquid nitrogen and stored at $-80^{\circ} \mathrm{C}$. The pellets were resuspended in cold lysis buffer $(1.85 \mathrm{M} \mathrm{NaOH}, 7.5 \%$ $\beta$-mercaptoethanol) to a final volume of $6 \mathrm{ml}$ and incubated for $15 \mathrm{~min}$ on ice. An equal volume of $55 \%$ trichloroacetic acid (TCA) was added, followed by incubation for $15 \mathrm{~min}$ on ice. Proteins were pelleted $(3.500 \mathrm{~g}, 5 \mathrm{~min}$, $4^{\circ} \mathrm{C}$ ), washed twice with cold water and resuspended in $12 \mathrm{ml}$ of buffer A and $0.05 \%$ Tween-20 (buffer A: $6 \mathrm{M}$ guanidinium chloride, $100 \mathrm{mM} \mathrm{NaH}_{2} \mathrm{PO}_{4}, 10 \mathrm{mM}$ Tris; $\mathrm{pH}$ 8.0). For resolubilzation, the suspensions were transferred into centrifuge tubes and incubated $(1 \mathrm{~h}, 180 \mathrm{rpm})$ while shaking. The clear supernatant was collected $(20 \mathrm{~min}$, $4^{\circ} \mathrm{C}, 23,000 \mathrm{~g}$ ), imidazole (final concentration $20 \mathrm{mM}$ ) and $200 \mu \mathrm{l}$ of Ni-NTA agarose slurry (Qiagen) were added to the tubes. The suspension was incubated overnight $\left(4^{\circ} \mathrm{C}\right)$. The agarose slurry was washed with $50 \mathrm{ml}$ of buffer A plus $0.05 \%$ Tween 20 , and $50 \mathrm{ml}$ of buffer C plus $0.05 \%$ Tween 20 (buffer C: $8 \mathrm{M}$ urea, $100 \mathrm{mM} \mathrm{NaH}_{2} \mathrm{PO}_{4}, 10 \mathrm{mM}$ Tris/HCl; pH 6.3). For protein elution, $1 \mathrm{ml}$ of elution buffer (buffer C, $0.05 \%$ Tween-20, $250 \mathrm{mM}$ imidazole) was added to the Ni-NTA column. The eluted proteins were precipitated with $1 \mathrm{ml}$ of $55 \%$ TCA, incubated for $10 \mathrm{~min}$ and centrifuged $(20,000 \mathrm{~g}, 30 \mathrm{~min})$. The pellet was boiled in $30 \mu \mathrm{HU}$-Buffer for $10 \mathrm{~min}$ at $65^{\circ} \mathrm{C}$.

Antibodies. Rpb1 antibodies 3E8 (Millipore), 3E10 (Millipore), 8WG16 (Abcam) and 4H8 (Abcam) were used. Anti-SUMO (Smt3) $)^{81}$ and Bur $1^{53}$ antibodies were described before. Anti-ubiquitin antibody P4D1 (Santa Cruz Biotechnology) and anti-Ctk1 (Abcam) were used. Anti-Dpm1 and anti-Pgk1 antibodies were purchased from Life Technologies.

Reproducibility. Rpb1 stability experiments with a western blot read-out in Fig. 1A were performed in four or more biological replicates. Quantification results in Fig. 1B were calculated from four biological replicates. Rpb1 stability experiments in Fig. 1C were performed in three biological replicates. In Fig. 1D, Rpb1 stability experiments in $c d c 48-6$ cells were performed three times, in $c d c 48-3$ cells once. Rpb1 stability experiments in Fig. $2 \mathrm{~A}$ were performed twice. In Fig. $2 \mathrm{~B}$, Rpb1 SUMOylation in $u b c 9-1$ cells was tested in two biological replicates, in siz1 $\Delta$ cells in four biological replicates, in siz2 $\Delta$ cells in two biological replicates and in siz1 $\Delta$ siz2 $\Delta$ cells in three biological replicates. Rpb1 stability experiments of Fig. 3A,B were performed in two and three biological replicates, respectively. Rpb1 stability experiments in Fig. 4A were performed in four or more biological replicates. Quantification results in Fig. 4B were calculated from four biological replicates. Experiments to detect Rpb1 ubiquitylation or SUMOylation in Fig. 4C,D were performed in three biological replicates. Denaturing Ni-PDs in Fig. $4 \mathrm{E}$ were performed in three biological replicates.

Received: 1 August 2019; Accepted: 30 October 2019;

Published online: 29 November 2019

\section{References}

1. Dammann, R., Lucchini, R., Koller, T. \& Sogo, J. M. Chromatin structures and transcription of rDNA in yeast Saccharomyces cerevisiae. Nucleic Acids Res. 21, 2331-2338 (1993).

2. Dieci, G., Conti, A., Pagano, A. \& Carnevali, D. Identification of RNA polymerase III-transcribed genes in eukaryotic genomes. Biochim. Biophys. Acta 1829, 296-305 (2013).

3. Neil, H. et al. Widespread bidirectional promoters are the major source of cryptic transcripts in yeast. Nature 457, 1038-1042 (2009).

4. van Dijk, E. L. et al. XUTs are a class of Xrn1-sensitive antisense regulatory non-coding RNA in yeast. Nature 475, 114-117 (2011). 
5. Tudek, A., Candelli, T. \& Libri, D. Non-coding transcription by RNA polymerase II in yeast: Hasard or nécessité? Biochimie 117, 28-36 (2015).

6. Schüller, R. et al. Heptad-Specific Phosphorylation of RNA Polymerase II CTD. Mol. Cell 61, 305-314 (2016).

7. Eick, D. \& Geyer, M. The RNA polymerase II carboxy-terminal domain (CTD) code. Chem. Rev. 113, 8456-8490 (2013).

8. Fong, N., Saldi, T., Sheridan, R. M., Cortazar, M. A. \& Bentley, D. L. RNA Pol II Dynamics Modulate Co-transcriptional Chromatin Modification, CTD Phosphorylation, and Transcriptional Direction. Mol. Cell 66, 546-557.e3 (2017).

9. Bataille, A. R. et al. A universal RNA polymerase II CTD cycle is orchestrated by complex interplays between kinase, phosphatase, and isomerase enzymes along genes. Mol. Cell 45, 158-170 (2012).

10. Suh, H., Hazelbaker, D. Z., Soares, L. M. \& Buratowski, S. The C-terminal domain of Rpb1 functions on other RNA polymerase II subunits. Mol. Cell 51, 850-858 (2013).

11. Kim, M., Suh, H., Cho, E.-J. \& Buratowski, S. Phosphorylation of the yeast Rpb1 C-terminal domain at serines 2, 5, and 7. J. Biol. Chem. 284, 26421-26426 (2009).

12. Mayer, A. et al. Uniform transitions of the general RNA polymerase II transcription complex. Nat Struct Mol Biol 17, 1272-1278 (2010).

13. Suh, H. et al. Direct Analysis of Phosphorylation Sites on the Rpb1 C-Terminal Domain of RNA Polymerase II. Mol. Cell 61, 297-304 (2016)

14. Spivak, G. Nucleotide excision repair in humans. DNA Repair (Amst.) 36, 13-18 (2015).

15. Gregersen, L. H. \& Svejstrup, J. Q. The Cellular Response to Transcription-Blocking DNA Damage. Trends Biochem. Sci. 43, 327-341 (2018).

16. Hara, R., Selby, C. P., Liu, M., Price, D. H. \& Sancar, A. Human transcription release factor 2 dissociates RNA polymerases I and II stalled at a cyclobutane thymine dimer. J. Biol. Chem. 274, 24779-24786 (1999).

17. Tremblay, M. et al. UV light-induced DNA lesions cause dissociation of yeast RNA polymerases-I and establishment of a specialized chromatin structure at rRNA genes. Nucleic Acids Res. 42, 380-395 (2014).

18. Chiou, Y.-Y., Hu, J., Sancar, A. \& Selby, C. P. RNA polymerase II is released from the DNA template during transcription-coupled repair in mammalian cells. J. Biol. Chem. 293, 2476-2486 (2018).

19. Cheung, A. C. M. \& Cramer, P. Structural basis of RNA polymerase II backtracking, arrest and reactivation. Nature 471, 249-253 (2011).

20. Epshtein, V. et al. UvrD facilitates DNA repair by pulling RNA polymerase backwards. Nature 505, 372-377 (2014).

21. Sigurdsson, S., Dirac-Svejstrup, A. B. \& Svejstrup, J. Q. Evidence that Transcript Cleavage Is Essential for RNA Polymerase II Transcription and Cell Viability. Mol. Cell 38, 202-210 (2010).

22. Donahue, B. A., Yin, S., Taylor, J. S., Reines, D. \& Hanawalt, P. C. Transcript cleavage by RNA polymerase II arrested by a cyclobutane pyrimidine dimer in the DNA template. PNAS 91, 8502-8506 (1994).

23. Wilson, M. D., Harreman, M. \& Svejstrup, J. Q. Ubiquitylation and degradation of elongating RNA polymerase II: the last resort. Biochim. Biophys. Acta 1829, 151-157 (2013).

24. Kuehner, J. N., Kaufman, J. W. \& Moore, C. Stimulation of RNA Polymerase II ubiquitination and degradation by yeast mRNA 3'end processing factors is a conserved DNA damage response in eukaryotes. DNA Repair (Amst.) 57, 151-160 (2017).

25. Geijer, M. E. \& Marteijn, J. A. What happens at the lesion does not stay at the lesion: Transcription-coupled nucleotide excision repair and the effects of DNA damage on transcription in cis and trans. DNA Repair (Amst.) 71, 56-68 (2018).

26. Ratner, J. N., Balasubramanian, B., Corden, J., Warren, S. L. \& Bregman, D. B. Ultraviolet radiation-induced ubiquitination and proteasomal degradation of the large subunit of RNA polymerase II. Implications for transcription-coupled DNA repair. J. Biol. Chem. 273, 5184-5189 (1998).

27. Beaudenon, S. L., Huacani, M. R., Wang, G., McDonnell, D. P. \& Huibregtse, J. M. Rsp5 ubiquitin-protein ligase mediates DNA damageinduced degradation of the large subunit of RNA polymerase II in Saccharomyces cerevisiae. Mol. Cell. Biol. 19, 6972-6979 (1999).

28. Huibregtse, J. M., Yang, J. C. \& Beaudenon, S. L. The large subunit of RNA polymerase II is a substrate of the Rsp 5 ubiquitin-protein ligase. PNAS 94, 3656-3661 (1997).

29. Mitsui, A. \& Sharp, P. A. Ubiquitination of RNA polymerase II large subunit signaled by phosphorylation of carboxyl-terminal domain. PNAS 96, 6054-6059 (1999).

30. Chang, A., Cheang, S., Espanel, X. \& Sudol, M. Rsp5 WW domains interact directly with the carboxyl-terminal domain of RNA polymerase II. J. Biol. Chem. 275, 20562-20571 (2000).

31. Somesh, B. P. et al. Communication between distant sites in RNA polymerase II through ubiquitylation factors and the polymerase CTD. Cell 129, 57-68 (2007).

32. Ribar, B., Prakash, L. \& Prakash, S. Requirement of ELC1 for RNA polymerase II polyubiquitylation and degradation in response to DNA damage in Saccharomyces cerevisiae. Mol. Cell. Biol. 26, 3999-4005 (2006).

33. Ribar, B., Prakash, L. \& Prakash, S. ELA1 and CUL3 are required along with ELC1 for RNA polymerase II polyubiquitylation and degradation in DNA-damaged yeast cells. Mol. Cell. Biol. 27, 3211-3216 (2007).

34. Harreman, M. et al. Distinct ubiquitin ligases act sequentially for RNA polymerase II polyubiquitylation. Proc. Natl. Acad. Sci. USA 106, 20705-20710 (2009)

35. Kvint, K. et al. Reversal of RNA polymerase II ubiquitylation by the ubiquitin protease Ubp3. Mol. Cell 30, 498-506 (2008).

36. Verma, R., Oania, R., Fang, R., Smith, G. T. \& Deshaies, R. J. Cdc48/p97 mediates UV-dependent turnover of RNA Pol II. Mol. Cell 41, 82-92 (2011)

37. Gillette, T. G., Gonzalez, F., Delahodde, A., Johnston, S. A. \& Kodadek, T. Physical and functional association of RNA polymerase II and the proteasome. Proc. Natl. Acad. Sci. USA 101, 5904-5909 (2004).

38. Silver, H. R., Nissley, J. A., Reed, S. H., Hou, Y.-M. \& Johnson, E. S. A role for SUMO in nucleotide excision repair. DNA Repair (Amst.) 10, 1243-1251 (2011)

39. Chen, X., Ding, B., LeJeune, D., Ruggiero, C. \& Li, S. Rpb1 sumoylation in response to UV radiation or transcriptional impairment in yeast. PLoS ONE 4, e5267 (2009).

40. Lafon, A. et al. INO80 Chromatin Remodeler Facilitates Release of RNA Polymerase II from Chromatin for Ubiquitin-Mediated Proteasomal Degradation. Mol. Cell 60, 784-796 (2015).

41. Chapman, R. D. et al. Transcribing RNA polymerase II is phosphorylated at CTD residue serine-7. Science 318, 1780-1782 (2007). 42. Lu, H. et al. Phase-separation mechanism for C-terminal hyperphosphorylation of RNA polymerase II. Nature 558, 318-323 (2018).

43. Woudstra, E. C. et al. A Rad26-Def1 complex coordinates repair and RNA pol II proteolysis in response to DNA damage. Nature 415, 929-933 (2002).

44. Ikenaga, M., Takebe, H. \& Ishii, Y. Excision repair of DNA base damage in human cells treated with the chemical carcinogen 4-nitroquinoline 1-oxide. Mutat. Res. 43, 415-427 (1977).

45. Rape, M. et al. Mobilization of processed, membrane-tethered SPT23 transcription factor by CDC48(UFD1/NPL4), a ubiquitinselective chaperone. Cell 107, 667-677 (2001).

46. Bergink, S. et al. Role of Cdc48/p97 as a SUMO-targeted segregase curbing Rad51-Rad52 interaction. Nat. Cell Biol. 15, 526-532 (2013).

47. Psakhye, I. \& Jentsch, S. Protein group modification and synergy in the SUMO pathway as exemplified in DNA repair. Cell 151, 807-820 (2012).

48. Albuquerque, C. P. et al. Distinct SUMO ligases cooperate with Esc2 and Slx5 to suppress duplication-mediated genome rearrangements. PLoS Genetics 9, e1003670 (2013). 
49. Jentsch, S. \& Psakhye, I. Control of nuclear activities by substrate-selective and protein-group SUMOylation. Annu. Rev. Genet. 47, $167-186$ (2013).

50. Cho, E. J., Kobor, M. S., Kim, M., Greenblatt, J. \& Buratowski, S. Opposing effects of Ctk1 kinase and Fcp1 phosphatase at Ser 2 of the RNA polymerase II C-terminal domain. Genes Dev. 15, 3319-3329 (2001).

51. Qiu, H., Hu, C. \& Hinnebusch, A. G. Phosphorylation of the Pol II CTD by KIN28 enhances BUR1/BUR2 recruitment and Ser2 CTD phosphorylation near promoters. Mol. Cell 33, 752-762 (2009).

52. Coordes, B. et al. Ctk1 function is necessary for full translation initiation activity in Saccharomyces cerevisiae. Eukaryotic Cell 14, $86-95$ (2015).

53. Clausing, E. et al. The transcription elongation factor Bur1-Bur2 interacts with replication protein A and maintains genome stability during replication stress. J. Biol. Chem. 285, 41665-41674 (2010).

54. Chymkowitch, P. et al. TORC1-dependent sumoylation of Rpc82 promotes RNA polymerase III assembly and activity. Proc. Natl. Acad. Sci. USA 114, 1039-1044 (2017).

55. Wang, Z., Wu, C., Aslanian, A., Yates, J. R. \& Hunter, T. Defective RNA polymerase III is negatively regulated by the SUMOUbiquitin-Cdc48 pathway. eLife 7 (2018).

56. Leśniewska, E., Cieśla, M. \& Boguta, M. Repression of yeast RNA polymerase III by stress leads to ubiquitylation and proteasomal degradation of its largest subunit, C160. BBA - Gene Regulatory Mechanisms 1862, 25-34 (2019).

57. Wang, Z. \& Prelich, G. Quality control of a transcriptional regulator by SUMO-targeted degradation. Mol. Cell. Biol. 29, 1694-1706 (2009).

58. Xie, Y., Rubenstein, E. M., Matt, T. \& Hochstrasser, M. SUMO-independent in vivo activity of a SUMO-targeted ubiquitin ligase toward a short-lived transcription factor. Genes Dev. 24, 893-903 (2010).

59. Schweiggert, J., Stevermann, L., Panigada, D., Kammerer, D. \& Liakopoulos, D. Regulation of a Spindle Positioning Factor at Kinetochores by SUMO-Targeted Ubiquitin Ligases. Dev. Cell 36, 415-427 (2016).

60. Höpfler, M. et al. Slx5/Slx8-dependent ubiquitin hotspots on chromatin contribute to stress tolerance. EMBO J. 38, e100368 (2019).

61. Malik, S., Bagla, S., Chaurasia, P., Duan, Z. \& Bhaumik, S. R. Elongating RNA polymerase II is disassembled through specific degradation of its largest but not other subunits in response to DNA damage in vivo. J. Biol. Chem. 283, 6897-6905 (2008).

62. Bowman, E. A. \& Kelly, W. G. RNA polymerase II transcription elongation and Pol II CTD Ser2 phosphorylation: A tail of two kinases. Nucleus 5, 224-236 (2014).

63. Wood, A., Schneider, J., Dover, J., Johnston, M. \& Shilatifard, A. The Bur1/Bur2 complex is required for histone H2B monoubiquitination by Rad6/Brel and histone methylation by COMPASS. Mol. Cell 20, 589-599 (2005).

64. Grenetier, S., Bouchoux, C. \& Goguel, V. CTD kinase I is required for the integrity of the rDNA tandem array. Nucleic Acids Res. 34, 4996-5006 (2006).

65. Liu, Y. et al. Phosphorylation of the transcription elongation factor Spt5 by yeast Bur1 kinase stimulates recruitment of the PAF complex. Mol. Cell. Biol. 29, 4852-4863 (2009).

66. Wyce, A. et al. H2B ubiquitylation acts as a barrier to Ctk1 nucleosomal recruitment prior to removal by Ubp8 within a SAGArelated complex. Mol. Cell 27, 275-288 (2007).

67. Ryu, H. Y. et al. The Ulp2 SUMOprotease promotes transcription elongation through regulation of histone sumoylation. EMBO J. 13, 67-19 (2019).

68. Yao, S. \& Prelich, G. Activation of the Bur1-Bur2 cyclin-dependent kinase complex by Cak1. Mol. Cell. Biol. 22, 6750-6758 (2002).

69. Ostapenko, D. \& Solomon, M. J. Phosphorylation by Cak1 regulates the C-terminal domain kinase Ctk1 in Saccharomyces cerevisiae. Mol. Cell. Biol. 25, 3906-3913 (2005).

70. Gwizdek, C. et al. The mRNA nuclear export factor Hprl is regulated by Rsp5-mediated ubiquitylation. J. Biol. Chem. 280, 13401-13405 (2005).

71. Iglesias, N. \& Stutz, F. Regulation of mRNP dynamics along the export pathway. FEBS Lett. 582, 1987-1996 (2008).

72. Gwizdek, C. et al. Ubiquitin-associated domain of Mex67 synchronizes recruitment of the mRNA export machinery with transcription. PNAS 103, 16376-16381 (2006)

73. Bretes, H. et al. Sumoylation of the THO complex regulates the biogenesis of a subset of mRNPs. Nucleic Acids Res. 42, 5043-5058 (2014).

74. Nie, M. et al. Dual recruitment of Cdc48 (p97)-Ufd1-Np14 ubiquitin-selective segregase by small ubiquitin-like modifier protein (SUMO) and ubiquitin in SUMO-targeted ubiquitin ligase-mediated genome stability functions. J. Biol. Chem. 287, 29610-29619 (2012).

75. Nagai, S. et al. Functional targeting of DNA damage to a nuclear pore-associated SUMO-dependent ubiquitin ligase. Science 322, 597-602 (2008)

76. Horigome, C. et al. PolySUMOylation by Siz 2 and Mms21 triggers relocation of DNA breaks to nuclear pores through the Slx5/Slx8 STUbL. Genes Dev. 30, 931-945 (2016).

77. Knop, M. et al. Epitope tagging of yeast genes using a PCR-based strategy: more tags and improved practical routines. Yeast 15, 963-972 (1999).

78. Janke, C. et al. A versatile toolbox for PCR-based tagging of yeast genes: new fluorescent proteins, more markers and promoter substitution cassettes. Yeast 21, 947-962 (2004).

79. Wang, G., Yang, J. \& Huibregtse, J. M. Functional domains of the Rsp5 ubiquitin-protein ligase. Mol. Cell. Biol. 19, 342-352 (1999).

80. Johnson, E. S. \& Blobel, G. Cell cycle-regulated attachment of the ubiquitin-related protein SUMO to the yeast septins. J Cell Biol 147, 981-994 (1999).

81. Hoege, C., Pfander, B., Moldovan, G.-L., Pyrowolakis, G. \& Jentsch, S. RAD6-dependent DNA repair is linked to modification of PCNA by ubiquitin and SUMO. Nature 419, 135-141 (2002).

82. Thompson, N. E., Steinberg, T. H., Aronson, D. B. \& Burgess, R. R. Inhibition of in vivo and in vitro transcription by monoclonal antibodies prepared against wheat germ RNA polymerase II that react with the heptapeptide repeat of eukaryotic RNA polymerase II. J. Biol. Chem. 264, 11511-11520 (1989).

83. Somesh, B. P. et al. Multiple mechanisms confining RNA polymerase II ubiquitylation to polymerases undergoing transcriptional arrest. Cell 121, 913-923 (2005)

\section{Acknowledgements}

We thank K. Sträßer for materials, and Sven Schkölziger, Alexander Strasser and Ulla Cramer for technical assistance. Ivan Psakhye for contributing SUMOylome data. Markus Höpfler, Claudio Lademann, Florian Paasch and members of the Jentsch and Pfander labs for critical discussion. Markus Höpfler, Claudio Lademann and Florian Paasch for critical reading of the manuscript. Research in the B.P. lab is supported by Max Planck Society, the collaborative research cluster (SFB1064) and project grants from the German Research Council (DFG). Research in the S.J. lab is supported by Max Planck Society, Deutsche Forschungsgemeinschaft, Center for Integrated Protein Science Munich, Louis-Jeantet Foundation and a European Research Council (ERC) Advanced Grant (\#339176). M.J.K. was supported by a fellowship of the Boehringer Ingelheim Fonds (BIF). 


\section{Author contributions}

I.H., M.K. and S.J. conceived the study. I.H. conducted all experiments. All authors contributed to the design of the experiments, analyzed the data and generated the concept of the paper, I.H. and B.P. wrote the manuscript, M.K. commented on the manuscript.

\section{Competing interests}

The authors declare no competing interests.

\section{Additional information}

Supplementary information is available for this paper at https://doi.org/10.1038/s41598-019-54027-y.

Correspondence and requests for materials should be addressed to B.P.

Reprints and permissions information is available at www.nature.com/reprints.

Publisher's note Springer Nature remains neutral with regard to jurisdictional claims in published maps and institutional affiliations.

(c) (i) Open Access This article is licensed under a Creative Commons Attribution 4.0 International License, which permits use, sharing, adaptation, distribution and reproduction in any medium or format, as long as you give appropriate credit to the original author(s) and the source, provide a link to the Creative Commons license, and indicate if changes were made. The images or other third party material in this article are included in the article's Creative Commons license, unless indicated otherwise in a credit line to the material. If material is not included in the article's Creative Commons license and your intended use is not permitted by statutory regulation or exceeds the permitted use, you will need to obtain permission directly from the copyright holder. To view a copy of this license, visit http://creativecommons.org/licenses/by/4.0/.

(c) The Author(s) 2019 